\title{
Sub-Micron Thin Film Intrinsic Josephson Junctions
}

\author{
P. A. Warburton, A. R. Kuzhakhmetov, C. Bell, G. Burnell, M. G. Blamire, H. Wu, C. R. M. Grovenor, and \\ H. Schneidewind
}

\begin{abstract}
We have fabricated sub-micron intrinsic Josephson junctions in thin films of Tl-Ba-Ca-Cu-O using two differing techniques suited to different applications. By using lateral focussed ion-beam milling we have created arrays of intrinsic junctions in $c$-axis oriented films. Such arrays, with areas as low as $0.25 \mu \mathrm{m}^{2}$, display large hysteresis comparable to that observed in single-crystal intrinsic junctions. By using normal focussed ion-beam milling we have created arrays in mis-aligned films grown on vicinal substrates. In arrays of area less than $0.4 \mu \mathrm{m}^{2}$ we observe Josephson phase diffusion and a suppressed critical current, showing that charging effects may be significant in these junctions.
\end{abstract}

Index Terms-Focussed ion-beam milling, Josephson junctions, nanofabrication.

\section{INTRODUCTION}

$\mathbf{S}$ INCE the spacing of consecutive copper-oxide double planes in many cuprate superconductors is greater than the coherence length in the $c$-direction, such planes are Josephson coupled. Hence a cuprate superconductor which is structured in such a way that the current is forced to flow in the $c$-direction acts as a series array of "intrinsic" Josephson junctions [1]. Such junctions show promise for a wide variety of applications. For example, zero-crossing Shapiro steps at frequencies up to $2.5 \mathrm{THz}$ have recently been measured in single crystal $\mathrm{Bi}-\mathrm{Sr}-\mathrm{Ca}-\mathrm{Cu}-\mathrm{O}$ intrinsic junctions [2], suggesting that they are suitable for development as voltage standards and sub-mm-wave oscillators. A variety of thin-film-based device geometries has also been proposed, with a view to further exploiting intrinsic Josephson effects in device applications; for a review of such devices see [3]. Here we report two techniques we have developed for fabrication of intrinsic Josephson junctions based on thin films of the superconductor $\mathrm{Tl}_{2} \mathrm{Ba}_{2} \mathrm{CaCu}_{2} \mathrm{O}_{8}$ (TBCCO). The two techniques, both of which make use of focussed ion-beam milling, allow us reliably to fabricate arrays of intrinsic junctions with sub-micron cross-sectional dimensions. It has been predicted [4], [5] that the charging energy is enhanced by a factor $N$ in capacitively coupled arrays of $N$ junctions. Since intrinsic junctions are strongly capacitively coupled, sub-micron intrinsic junctions may show charging effects at temperatures of $4.2 \mathrm{~K}$ or higher.

Manuscript received August 6, 2002. This work was supported in part by the U.K. Engineering and Physical Sciences Research Council.

P. A. Warburton and A. R. Kuzhakhmetov are with University College London, Department of Electrical and Electronic Engineering, Torrington Place, London WC1E 7JE, U.K. (e-mail: p.warburton@ee.ucl.ac.uk).

G. Burnell, C. Bell and M. G. Blamire are with University of Cambridge, Department of Materials Science, Cambridge, CB2 3QZ, U.K.

H. Wu and C. R. M. Grovenor are with University of Oxford, Department of Materials, Parks Road, Oxford OX1 3PH, U.K

H. Schneidewind is with IPHT-Jena, 07745 Jena, Germany.

Digital Object Identifier 10.1109/TASC.2003.814056

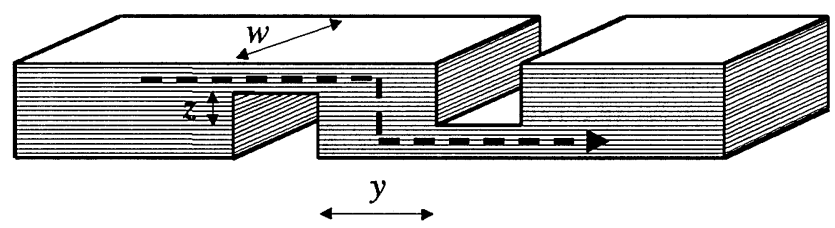

(a)

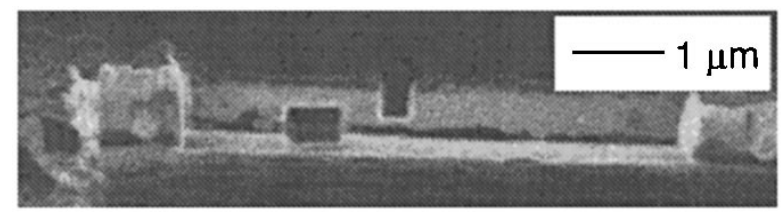

(b)

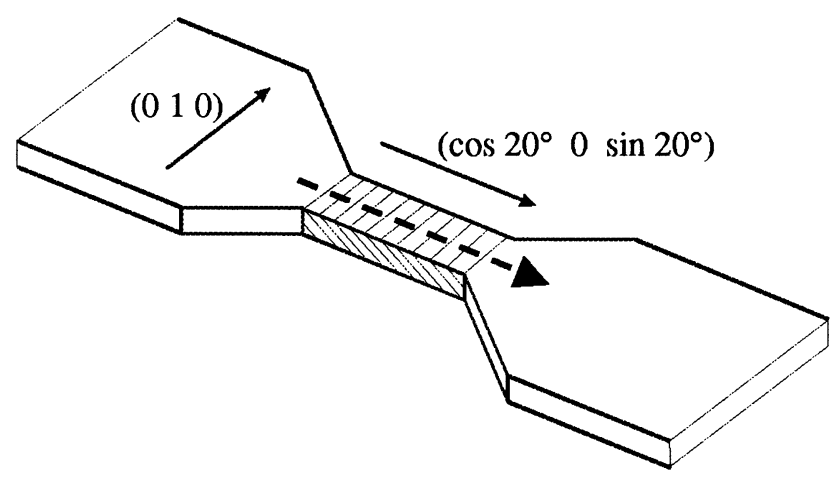

(c)

Fig. 1. (a) Schematic (not to scale) of device geometry utilising $c$-axis oriented film. The horizontal lines in the film represent the copper-oxide double planes. The dotted line represents the transport current. Typical device dimensions are: $w=0.7 \mu \mathrm{m} ; y=1 \mu \mathrm{m} ; z=0.1 \mu \mathrm{m}$. (b) Scanning-ion micrograph of a device fabricated in a $c$-axis oriented film. The angle between the ion-beam and the substrate surface is set at $5^{\circ}$ so as to show the side view of the device. (c) Schematic (not to scale) of device geometry utilising a mis-aligned film. The slanting lines in the bridge represent the copper-oxide double planes. The dotted line represents the transport current.

In addition to the sub-mm-wave applications referred to above, our junctions may therefore be developed into single electron device applications [6].

\section{DEVICE FABRICATION}

\section{A. c-Axis Devices}

Our device geometry which makes use of $c$-axis oriented TBCCO films is shown in Fig. 1(a). This structure is based on a similar device fabricated in $\mathrm{Bi}-\mathrm{Sr}-\mathrm{Ca}-\mathrm{Cu}-\mathrm{O}$ whiskers by $\mathrm{Kim}$ et al. [7]. The films, of typical thickness $800 \mathrm{~nm}$, are grown on ( $\left.\begin{array}{lll}0 & 0 & 1\end{array}\right)$ oriented lanthanum aluminate single crystal substrates in a two-stage process. An amorphous sputtered $\mathrm{Ba}-\mathrm{Ca}-\mathrm{Cu}-\mathrm{O}$ precursor is annealed ex situ in a thallous atmosphere at around $850^{\circ} \mathrm{C}$ [8]. The film is encapsulated by a sputtered gold film of thickness $300 \mathrm{~nm}$. 
Gross patterning of the TBCCO lines which contact to the devices is done by using optical lithography and argon ion-milling. Tracks of typical width $w=0.7 \mu \mathrm{m}$ are then patterned in the film using normally-incident focussed ion-beam milling with a $30 \mathrm{keV}$ gallium ion-beam. The substrate is then rotated so that the ion beam is almost parallel to it - in fact the angle between the beam and the substrate is typically $5^{\circ}$. This allows us to mill laterally the slots which force the current to flow parallel to the $c$-axis in the central part of the structure. Hence we have a stack of intrinsic junctions of cross-sectional area $w y$ and stack height $z$, which is expected to contain $N=(z / 1.7 \mathrm{~nm})$ intrinsic junctions. A scanning ion micrograph of the final structure is shown in Fig. 1(b).

\section{B. Mis-Aligned Devices}

Our device geometry which makes use of mis-aligned TBCCO films is shown in Fig. 1(c). The films, of typical thickness $250 \mathrm{~nm}$ are grown by the same process as for our $c$-axis films. Here, however, the substrate is vicinal lanthanum aluminate with its surface cut at an angle of $20^{\circ}$ to the $\left(\begin{array}{lll}0 & 0 & 1\end{array}\right)$ axis. During the annealing process the TBCCO film recrystallises epitaxially on the vicinal substrate with the result that the copper-oxide double planes are mis-aligned by $20^{\circ}$ with respect to the substrate surface. This epitaxy is confirmed both by four-circle $\mathrm{x}$-ray diffraction and cross-sectional transmission electron micrography [9].

\section{RESULTS}

\section{A. Current-Voltage Characteristics}

The current - voltage characteristics were obtained by mounting the devices on a copper block at the end of a probe which was immersed in helium vapor in a storage dewar. Mini-coaxial cables run along the length of the probe to connect the sample, via $L C$ filters with cut-off frequency 200 $\mathrm{Hz}$, to room-temperature electronics. This consists of a current source which is swept quasistatically at between $1 \mathrm{~Hz}$ and 20 $\mathrm{Hz}$, and a low-noise voltage amplifier.

All our devices show multi-branched current - voltage characteristics. A typical example, taken from a device in a $c$-axis oriented film, is shown in Fig. 2. There is a supercurrent branch at low (not always zero) voltage and $N$ quasiparticle branches. Here $N=94 \pm 2$. This branched structure confirms the existence of a multitude of Josephson junctions in the array and is consistent with the depth of the stack, $z$ (see Fig. 1), and the copper-oxide double plane spatial periodicity of $1.7 \mathrm{~nm}$. The nearly vertical part of the characteristics at a voltage of $2.44 \mathrm{~V}$ corresponds to all $N$ junctions being at the gap voltage, $2 \Delta / e$. Hence we can estimate $2 \Delta=26 \mathrm{meV}$, in good agreement with other tunnelling measurements of the gap voltage in TBCCO [10]. The small value of the ratio of the retrapping current to the critical current shows that the Stewart - McCumber parameter, $\beta_{c}$, is large $\left(\sim 310^{3}\right)$. This in turn suggests that dissipation is low and comparable with the level of dissipation in intrinsic junctions based on single crystals.

Features in the quasiparticle branches labeled with an arrow in Fig. 2 occur due to Josephson oscillations at the same frequency as $c$-axis optical phonon modes in the TBCCO structure

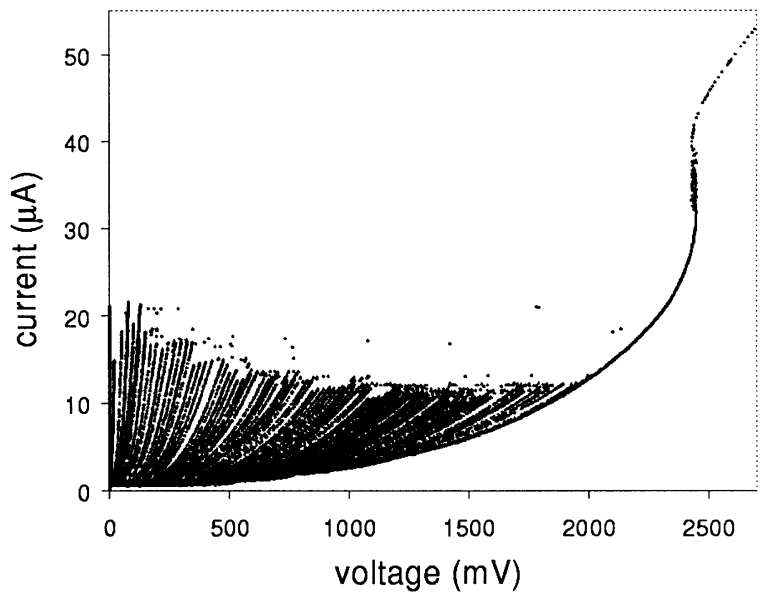

(a)

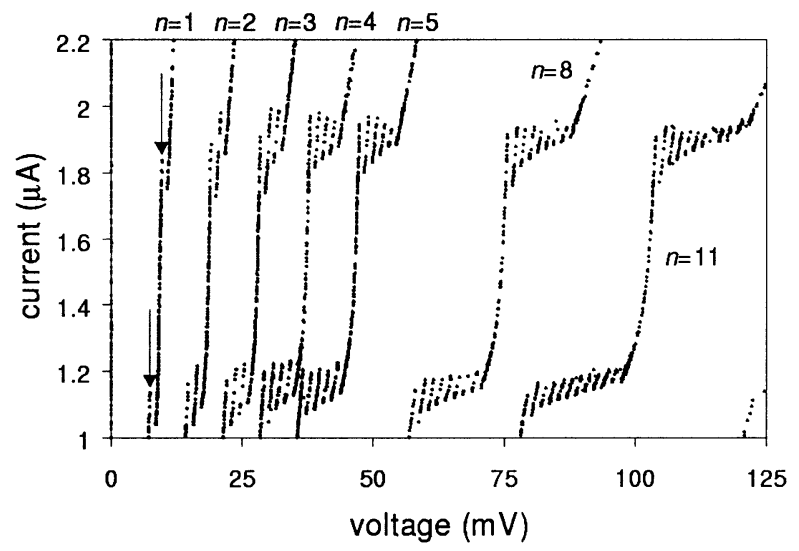

(b)

Fig. 2. (a) $4.2 \mathrm{~K}$ current-voltage characteristics of a device fabricated in a $c$-axis film. (b) is the enlargement of the low-voltage low-current region of (a), showing some of the first 11 quasiparticle branches. The arrows show the phonon resonances in the first quasiparticle branch (see text).

[11]. The splitting of the features into $n$ on the $n$th quasiparticle branch is clearly visible for $n$ up to 11 . To our knowledge such clear resonances at large $n$ have not previously been observed in other thin film intrinsic junction types, probably since they are damped out. This further confirms that our devices (at least in $c$-axis films) are lightly damped, as is the case for single crystal intrinsic junctions. We conclude therefore that for intrinsic junction applications our $c$-axis films are of comparable quality to single crystals of anisotropic superconductors.

\section{B. Area Dependence of the Switching Current}

The dependence of the switching current density at $4.2 \mathrm{~K}$ on the device cross-sectional area is shown in Fig. 3. Here the switching current is defined as the value of an increasing quasistatic current bias at which the jump from the supercurrent branch to the first quasiparticle branch occurs. For devices of area greater than $0.8 \mu \mathrm{m}^{2}$ we measure a broad distribution of switching currents over repeated measurements [12]; the data points here correspond to the peak switching current in the distribution. Note that the data for the two types of device geometry overlap, confirming that the $a b$-plane transport currents present in the mis-aligned devices have a negligible effect on the $c$-axis Josephson coupling. 


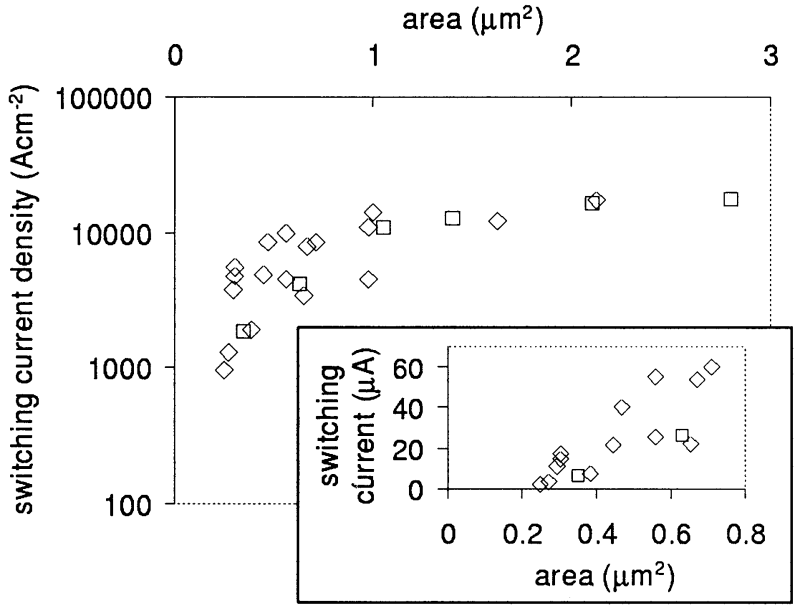

Fig. 3. Dependence of the switching current density (on a logarithmic scale) at a temperature of $4.2 \mathrm{~K}$ on the cross-sectional junction area. The inset shows the dependence of the switching current at $4.2 \mathrm{~K}$ on area for our smallest devices. In both graphs the squares are data from a single mis-aligned device whose area was sequentially reduced by normal focussed ion-beam milling. The diamonds are data from a series of $c$-axis devices fabricated in two different TBCCO films

For junctions of area greater than $1 \mu \mathrm{m}^{2}$ the switching current density is essentially constant at $1.7 \pm 0.410^{4} \mathrm{Acm}^{-2}$. If we take this to be the critical current density of the intrinsic junctions we estimate a plasma frequency of $470 \mathrm{GHz}$. (This estimate is made by treating the intrinsic junctions as parallel plate capacitors with dielectric thickness $1.5 \mathrm{~nm}$ and relative permittivity 10.) This is within a factor 2 of that measured by infra-red spectroscopy of TBCCO films [13].

For junctions of area less than $1 \mu \mathrm{m}^{2}$ we observe a rapid suppression of the switching current density which can be extrapolated to zero at an area of $0.2 \mu \mathrm{m}^{2}$. Similar reductions of the switching current density have been observed in sub-micron $\mathrm{Bi}-\mathrm{Sr}-\mathrm{Ca}-\mathrm{Cu}-\mathrm{O}$ single crystal intrinsic junctions [14], [15] and may be attributable to part of the junction area being damaged during fabrication and/or a fundamental change in the device physics at short lengthscales. Since this reduction in switching current density is also accompanied by (a) elimination of strong fluctuations for junctions of area less than $0.8 \mu \mathrm{m}^{2}$ and (b) the appearance of dissipation at currents less than the switching current for junctions of area less than $0.4 \mu \mathrm{m}^{2}$ (see below), it is likely that the physics which determine the junction transport properties are modified for our small area junctions. On the other hand, extrapolation from the critical current density of our large area junctions suggests that the Josephson energy, $E_{J}$, of a junction of area $0.2 \mu \mathrm{m}^{2}$ is $810 k \mathrm{~K}$, where $k$ is Boltzmann's constant. It is difficult to understand how the device properties can be so strongly modified at operating temperatures $T \ll E_{J} / k$. We conclude, therefore, that both junction damage during fabrication (most likely due to implantation of the edges of the superconducting film by gallium ions) and unconventional junction physics play roles in modifying the transport properties of our sub-micron intrinsic Josephson junctions.

\section{Phase Diffusion}

The supercurrent branch of junctions of area less than 0.4 $\mu \mathrm{m}^{2}$ is not at zero voltage - i.e., dissipation occurs for bias currents less than the switching current. The form of the quasipar-

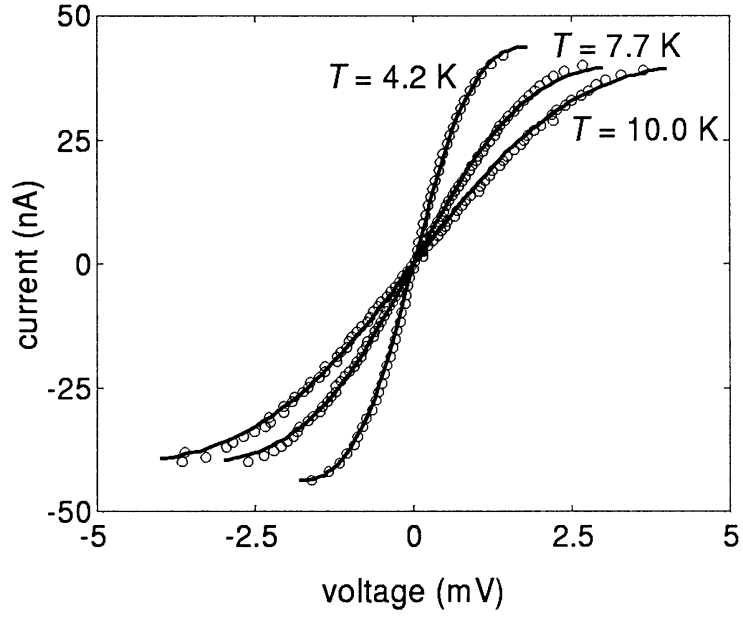

Fig. 4. Current - voltage characteristics of a mis-aligned device. Only the supercurrent branch is shown. The points are experimental data. The lines are the prediction of the theory of Ingold et al. (see text) with the impedance $Z$ seen by the junction as the only free parameter. From the fits, $Z$ is $10.2 \mathrm{k} \Omega, 10.5 \mathrm{k} \Omega$ and $11.5 \mathrm{k} \Omega$ at $4.2 \mathrm{~K}, 7.7 \mathrm{~K}$, and $10 \mathrm{~K}$ respectively.

ticle branches of these devices are, however, identical to those of larger junctions, and the characteristics remain strongly hysteretic. This dissipative branch is observed both in junctions with $E_{J}>k T$ [16] and $E_{J}<k T$. A typical example of the latter is shown in Fig. 4. Note that this junction has an anomalously low switching current density $\left(\sim 30 \mathrm{Acm}^{-2}\right.$ at $\left.4.2 \mathrm{~K}\right)$, probably due to damage during ion milling. The form of the supercurrent branch is similar to that previously observed in small hysteretic low $T_{c}$ junctions and attributed to phase diffusion [17]. The data fit very well to the theory of phase diffusion by Ingold et al. [18]. We use only one free parameter, this being the impedance seen by the junction, equal here to $10.9 \pm 0.7 \mathrm{k} \Omega$. The fit to the data is excellent up to a temperature of $10 \mathrm{~K}$, at which point the hysteresis in the data vanishes, making comparison with the theory impossible.

While phase diffusion, being a random process, is not advantageous for applications, study of the phase diffusion branch of ultra-small junctions can reveal information on the charging energy in such junctions. Since this parameter is of fundamental importance for understanding the device physics and may be exploited in single electron device applications, further studies of phase diffusion in our junctions are ongoing.

\section{CONCLUSIONS}

We have developed two distinct techniques for fabricating sub-micron intrinsic Josephson junctions. The observation of a phase diffusion branch at currents less than the switching current and suppression of the critical current for small area junctions suggest that charging effects may be significant in these devices. In order to exploit such effects it will be necessary to control the electrostatic potential of the junctions by applying a voltage to a gate electrode. The mis-aligned devices in vicinal films are suited to experiments in which the gate electrode is on top of the bridge. These devices are, however, not suited to experiments in which precise control of the number of junctions is required. We are currently investigating the possibility of this by monitoring the conductance of our $c$-axis devices during the ion-beam milling process. 


\section{REFERENCES}

[1] R. Kleiner, F. Steinmeyer, G. Kunkel, and P. Mueller, "Intrinsic Josephson effects in $\mathrm{Bi}_{2} \mathrm{Sr}_{2} \mathrm{CaCu}_{2} \mathrm{O}_{8}$ single-crystals," Phys. Rev. Lett., vol. 68, p. 2394, 1992.

[2] H. B. Wang, P. H. Wu, and T. Yamashita, "Terahertz responses of intrinsic Josephson junctions in high $T_{c}$ superconductors," Phys. Rev. Lett., vol. 87, p. 107 002, 2001.

[3] A. A. Yurgens, "Intrinsic Josephson junctions: Recent developments," Supercond. Sci. Technol., vol. 13, p. R85, 2000.

[4] K. K. Likharev, N. S. Bakhvalov, G. S. Kazacha, and S. I. Serdyukava, "Single-electron tunnel junction array: An electrostatic anolog of the Josephson transmission line," IEEE Trans. Mag., vol. 25, p. 1436, 1989.

[5] K. K. Likharev and K. A. Matsuoka, "Electron-electron interaction in linear arrays of small tunnel junctions," Appl. Phys. Lett., vol. 67, p. 3037, 1995.

[6] K. K. Likharev, "Single-electron devices and their applications," Proc. IEEE, vol. 87, p. 606, 1999.

[7] S. J. Kim, Y. I. Latyshev, and T. Yamashita, "Submicron stacked-junction fabrication from $\mathrm{Bi}_{2} \mathrm{Sr}_{2} \mathrm{CaCu}_{2} \mathrm{O}_{8+\delta}$ whiskers by focused-ion-beam etching," Appl. Phys. Lett., vol. 74, p. 1156, 1999.

[8] A. P. Bramley, J. D. O'Connor, and C. R. M. Grovenor, "Thallium-based HTS thin films, processing, properties and applications," Supercond. Sci. Technol., vol. 12, p. R57, 1999.

[9] O. S. Chana, A. R. Kuzhakhmetov, D. M. C. Hyland, C. J. Eastell, D. Dew-Hughes, C. R. M. Grovenor, Y. Koval, M. Moessle, R. Kleiner, P. Müller, and P. A. Warburton, "Fabrication and measurement of intrinsic Josephson junctions in misaligned films of $\mathrm{Tl}_{2} \mathrm{Ba}_{2} \mathrm{CaCu}_{2} \mathrm{O}_{8}$," IEEE Trans. Appl. Supercond., vol. 11, p. 2711, 2001.

[10] I. Takeuchi, J. Tsai, Y. Shimakawa, T. Manako, and Y. Kubo, "Energy-gap of Tl-Ba-Ca-Cu-O compounds by tunneling," Physica C, vol. 158 , p. 83, 1989.
[11] K. Schlenga, R. Kleiner, G. Hechtfischer, M. Moessle, S. Schmitt, P. Mueller, C. Helm, C. Preis, F. Forsthofer, J. Keller, H. L. Johnson, M. Veith, and E. Steinbeiss, "Tunneling spectroscopy with intrinsic Josephson junctions in BSCCO and TBCCO," Phys. Rev. B, vol. 57, p. $14518,1998$.

[12] P. A. Warburton, A. R. Kuzhakhmetov, O. S. Chana, D. M. C. Hyland, C. R. M. Grovenor, G. Burnell, M. G. Blamire, and H. Schneidewind, "Multiple-peak switching current distribution in Tl-Ba-Ca-Cu-O intrinsic Josephson junctions," Physica C, to be published.

[13] D. Dulic, D. van der Marel, A. A. Tsvetkov, W. N. Hardy, Z. F. Ren, J. H. Wang, and B. A. Willemsen, " $c$-axis penetration depth and interlayer conductivity in the thallium-based cuprate superconductors," Phys. Rev. $B$, vol. 60, p. R15051, 1999.

[14] A. Irie, G. Oya, R. Kleiner, and P. Mueller, "Transport properties of small-sized intrinsic Josephson junctions in Bi-Sr-Ca-Cu-O," Physica $C$, vol. 362, p. 145, 2001.

[15] S. J. Kim, Y. I. Latyshev, T. Yamashita, and S. Kishida, "New approach for fabricating submicron scale intrinsic Josephson junctions using high- $T_{c}$ superconducting materials," Physica $C$, vol. 362, p. 150, 2001.

[16] P. A. Warburton, A. R. Kuzhakhmetov, G. Burnell, M. G. Blamire, and H. Schneidewind, "Decoupling of a small current-biased Josephson junction from its environment," Phys. Rev. Lett., submitted for publication.

[17] J. M. Martinis and R. L. Kautz, "Classical phase diffusion in small hysteretic Josephson junctions," Phys. Rev. Lett., vol. 63, p. 1507, 1989.

[18] G.-L. Ingold, H. Grabert, and U. Eberhardt, "Cooper-pair current through ultrasmall Josephson junctions," Phys. Rev. B, vol. 50, p. 395, 1994 